\title{
Soft Tissue Infection
}

National Cancer Institute

\section{Source}

National Cancer Institute. Soft Tissue Infection. NCI Thesaurus. Code C79745.

A bacterial infectious process affecting the soft tissues. A severe form caused by anaerobic or aerobic bacteria is associated with soft tissues necrosis and affects the subcutaneous adipose tissue, muscles, fascia, and the skin. 